\title{
A Novel Non-Iterative Parameter Estimation Method for Interval Type-2 Fuzzy Neural Networks Based on a Dynamic Cost Function
}

\author{
Mojtaba Ahmadieh Khanesar ${ }^{1}$, Saima Hassan ${ }^{2}$ and Erik Cambria ${ }^{3}$, Erdal Kayacan ${ }^{4}$
}

\begin{abstract}
Non-iterative methods for parameter estimation for interval type-2 neuro-fuzzy structure are fast to implement, when compared to online methods, and need no -or a fewparameters to be tuned. In this paper, a novel dynamic cost function, which defines a relationship between the current and past errors, is defined. The minimization of the aforementioned cost function results in a decreasing sequence of error which makes the proposed method numerically more stable when compared to least squares-based methods. It is a well-known phenomenon that a matrix inversion may cause problems if the matrix to be inverted is ill-defined i.e. its condition number is far bigger than one. The use of a dynamic relationship between the current and past error adds more degrees of freedom which makes it possible to improve the condition number of the matrix. Comprehensive simulation studies are presented for the prediction of financial data sets. The simulation results shows the superior numerical stability of the proposed method as the mean value of the condition number is smaller. This finding results in more accurate matrix inversion to be done in the two-step matrix inversion.
\end{abstract}

\section{INTRODUCTION}

Interval type-2 fuzzy logic systems (IT2FLSs) become well-known systems for their ability to deal with uncertainty, noisy and incomplete data. IT2FLSs benefit from type-2 membership functions (MFs) with fuzzy membership grades [1]. This structure demonstrated superior performance over their type-1 counterparts specially when high levels of noise and uncertainties exist in the system [2]-[5].

Among various learning algorithms, the Gradient descentbased algorithms have been dominantly utilized for training the neural networks (NNs) [6]. The back-propagation (BP) algorithms and its variants suffer from various learning issues including stop criteria, number of learning epoch and the possibility of entrapment in a local minimum during training of NNs [7] and [8]. Such issues have emerged novel noniterative learning schemes [9]. Various least squares (LS) based methods are proposed to solve the limitations of BP algorithms and has shown its efficiency in training the NNs [10]. This method is a non-iterative method and makes it possible to find the results in two iterations. The fact

\footnotetext{
*This work was not supported by any organization

${ }^{1}$ M. Ahmadieh Khanesar is with Faculty of Engineering, Mechaincal, Materials and Manufacturing Department, University of Nottingham, NG7 2RD Nottingham, UK ezzma5 eexmail.nottingham.ac.uk

${ }^{2}$ Saima Hassan is with Institute of Information Technology, Kohat University of Science and Technology, Kohat, KPK, Pakistan saimahassan@kust.edu.pk

${ }^{4}$ Erik Cambria is with the School of Computer Science and Engineering, Nanyang Technological University, Singapore cambria@ntu.edu.sg

${ }^{2}$ Erdal Kayacan is with Aarhus University, Department of Engineering, Aarhus C, DK-8000, Denmark erdal@eng.au.dk
}

that there is no need to tune any parameters, makes this algorithm a very fast algorithm which does not necessitate any iterations to select its parameters.

The LS based method in [8] can be considered as a feed-forward NN (FFNN) where learning of the system takes place in two stages. In the first stage, the weights connecting input layer and the hiddden layer are selected randomly [11], [12]. In the second stage, the connections with output neurons (weights of output layer) are analytically determined. This makes LS training of FFNN as a linear learning problem where only the connections with output neurons need to be adjusted. Solving a linear system with the random parameters reduces computation time than that of the iterative BP algorithms without sacrificing the prediction accuracy. The efficacy of this method over the support vector machines and its variants has been empirically shown [13]. Other variants of non-iterative learning algorithms are evolutionary ELM [7], optimally pruning ELM (OPELM) [14], and bidirectional ELM (B-ELM) [15] that tries decreases the number of hidden nodes without affecting learning effectiveness of the algorithm. In these algorithms the optimal nodes are selected in a way that the ones with lower residual error among the several randomly generated hidden nodes.

In this paper, an innovative non-iterative training algorithm to tune the parameters of IT2FLSs is introduced. Although previous non-iterative optimization methods are based on the minimization of sum of squared error, in the proposed method, the cost function is sum of squared of a discrete time dynamic relationship in the form of $s(t)=e(t)+$ $\lambda e(t-1),|\lambda|<1$. Such cost function has been previously used in [16], but not solved using non-iterative approaches. Since absolute value of the parameter $\lambda$ is less than one, minimization of the sum of squares results in decreasing error with respect to time. Although, it can be proved that existing non-iterative training methods can optimally tune the consequent part parameters of IT2FLSs, the proposed method shows superior performance with the test data. This is mainly because of the numerical stability caused by the minimization of dynamic relationship. One of the main challenges with existing non-iterative optimization is that they include matrix inversion which may cause numerical instability. However, the minimization of the proposed cost function which is based on the current and past error improves the condition number of the matrices to be inverted. The condition number in this case depends on the selection of the parameter $\lambda$ which adds more degrees of freedom to the optimization method. To test the performance of the proposed training 
method in dealing with real time datasets, it is applied on the prediction of SGD/USD and EURO/USD exchange rates. The simulation results show superior performance for the test data when compared to the existing non-iterative algorithms for training of the IT2FLSs.

This paper is organized as follows. The structure of IT2FLSs and existing non-iterative optimization methods are introduced in Section II. In Section III, the proposed noniterative optimization method based on proposed dynamic cost function is discussed. Simulation results are presented in Section IV. The concluding marks are presented in Section V.

\section{Structure of the Interval Type 2 FuZzy Logic SYSTEM}

Type-2 fuzzy MFs benefit from a secondary MFs which make the membership grade fuzzy. The fuzzy system, which is constructed using this type of MFs, are called generalized type-2 fuzzy logic systems. However, if the secondary membership grades are considered as either equal to zero or one, the resulting fuzzy system is called IT2FLS. This simplification makes it possible for IT2FLSs to be executed much faster than the general IT2FLSs. The main difference between T2FLSs and T1FLSs is that in IT2FLS output processing block which consists of a type-reducer followed by a defuzzifier block replaces defuzziffier block of T1FLSs (see Fig. 1).

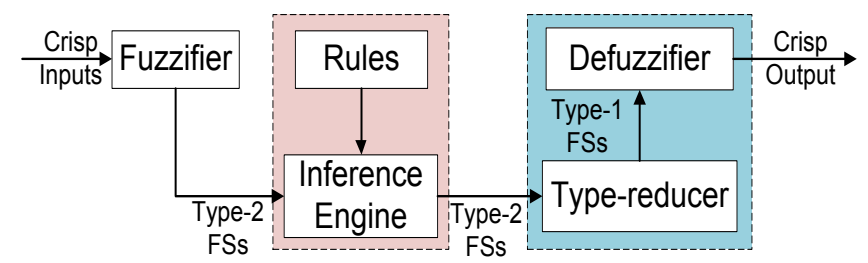

Fig. 1: Structure of the IT2FLS.

A generalized IF-THEN rule $\left(R^{n}\right)$ with $N$ number of rules for an IT2FLS is as follows [17]:

$R^{n}:$ IF $x_{1}$ is $\tilde{X}_{1}^{n} \wedge x_{2}$ is $\tilde{X}_{2}^{n} \wedge \cdots \wedge x_{d}$ is $\tilde{X}_{d}^{n}$

THEN $y^{n}(\mathbf{x})=c_{0}^{n}+c_{1}^{n} x_{1}+\cdots+c_{d}^{n} x_{d}, \quad n=1, \cdots, N$

The input to each fuzzy rule a vector of $d$-dimensional input $\mathbf{x}=\left[x_{1}, x_{2}, \cdots, x_{d}\right]^{T}$, which maps the fuzzy set to a varying singleton $y^{n} . \tilde{X}_{i}^{n}, i=1 \cdots, d$, are the $i_{t h}$ interval type-2 fuzzy subset generated from the input variable $x_{i}$ in the $n_{t h}$ rule domain with $N$ being the total number of fuzzy rules. $\mathbf{c}^{n}=\left[c_{0}^{n}, c_{1}^{n}, \cdots, c_{d}^{n}\right]^{T}$ represents the parameters of $n_{t h}$ fuzzy rule.

Since Gaussian MF are sufficiently smooth functions which are one of the most frequently used fuzzy MFs. However, in the case an interval value is considered for the center of the Gaussian MF, the resulting upper and lower Gaussian MFs are not differentiable. Such Gaussian type$2 \mathrm{MF}$ with a fixed $\sigma_{i}^{n}$ parameter and an interval center value that takes on values in $\left[m_{i}^{n 1}, m_{i}^{n 2}\right],[18]$ and [17] is as follows:

$$
\mu_{\tilde{X}_{i}^{n}}\left(x_{i}\right)=\exp \left[-\frac{1}{2}\left(\frac{x_{i}-m_{i}^{n}}{\sigma_{i}^{n}}\right)^{2}\right], \quad m_{i}^{n} \in\left[m_{i}^{n 1}, m_{i}^{n 2}\right]
$$

The type-2 fuzzy MF $\mu_{\tilde{X}_{i}^{n}}\left(x_{i}\right)$ of (1) has upper fuzzy MF, $\left.\bar{\mu}_{\tilde{X}_{i}^{n}}\left(x_{i}\right)\right)$, and lower fuzzy MF $\underline{\mu}_{\tilde{X}_{i}^{n}}\left(x_{i}\right)$, as follows:

$$
\begin{array}{r}
\bar{\mu}_{\tilde{X}_{i}^{n}}\left(x_{i}\right)=\left\{\begin{array}{cc}
N\left(m_{i}^{n 1}, \sigma_{i}^{n} ; x_{i}\right), & x_{i}<m_{i}^{n 1} \\
1, & m_{i}^{n 1} \leq x_{i} \leq m_{i}^{n 2} \\
N\left(m_{i}^{n 2}, \sigma_{i}^{n} ; x_{i}\right), & x_{i}>m_{i}^{n 2}
\end{array}\right. \\
\underline{\mu}_{\tilde{X}_{i}^{n}}\left(x_{i}\right)=\left\{\begin{array}{cc}
N\left(m_{i}^{n 2}, \sigma_{i}^{n} ; x_{i}\right), & x_{i} \leq \frac{m_{i}^{n 1}+m_{i}^{n 2}}{2} \\
N\left(m_{i}^{n 1}, \sigma_{i}^{n} ; x_{i}\right), & x_{i}>\frac{m_{i}^{n 1}+m_{i}^{n 2}}{2}
\end{array}\right.
\end{array}
$$

The firing strength of a rule corresponding to an input set is an interval value, $F^{n}=\left[\underline{f}^{n}, \bar{f}^{n}\right]$, where

$\underline{f}^{n}(x)=\underline{\mu}_{\tilde{X}_{1}^{n}}\left(x_{1}\right) \star \underline{\mu}_{\tilde{X}_{2}^{n}}\left(x_{2}\right) \star \cdots \star \underline{\mu}_{\tilde{X}_{d}^{n}}\left(x_{d}\right)=\prod_{i=1}^{d} \underline{\mu}_{\tilde{X}_{i}^{n}}\left(x_{i}\right)$

and

$\bar{f}^{n}(x)=\bar{\mu}_{\tilde{X}_{1}^{n}}\left(x_{1}\right) \star \bar{\mu}_{\tilde{X}_{2}^{n}}\left(x_{2}\right) \star \cdots \star \bar{\mu}_{\tilde{X}_{d}^{n}}\left(x_{d}\right)=\prod_{i=1}^{d} \bar{\mu}_{\tilde{X}_{i}^{n}}\left(x_{i}\right)$

The output of the IT2FLS is obtained as follows:

$$
\begin{aligned}
Y= & {\left[y_{l}, y_{r}\right] } \\
= & \int_{y^{1} \in\left[\underline{y}_{1}, \bar{y}_{1}\right]} \cdots \int_{y^{N} \in\left[\underline{y}_{N}, \bar{y}_{N}\right]} \\
& \int_{f_{1} \in\left[\underline{f}_{1}, \bar{f}_{1}\right]} \cdots \int_{f_{N} \in\left[\underline{f}_{N}, \bar{f}_{N}\right]} 1 / \frac{\sum_{n=1}^{N} f_{n} y^{n}}{\sum_{n=1}^{N} f_{n}}
\end{aligned}
$$

where:

$$
y^{n}=c_{0}^{n}+\sum_{i=1}^{d} c_{i}^{n} x_{i}=\sum_{i=0}^{d} c_{i}^{n} x_{i}
$$

and $x_{0}=1$.

The indices $l$ and $r$ in the IT2 fuzzy sets $\left[y_{l}, y_{r}\right]$ represent left and right limits, respectively. There exists different approaches to calculate the outputs of IT2FLSs [19], among which Enhanced Karnik-Mendel (EKM) method [20] is used because of its efficacy and most frequently usage. Assume $\mathbf{y}=\left[y^{1}, \cdots, y^{N}\right]^{T}$ as the original rule-ordered consequent values, and $\tilde{\mathbf{y}}=\left[\tilde{y}^{1}, \cdots, \tilde{y}^{N}\right]^{T}$ as the reordered consequent values, where $\tilde{y}^{1} \leq \tilde{y}^{2} \leq \cdots \leq \tilde{y}^{N}$. The parameters $f_{n}$ and $\bar{f}_{n}$ are also reordered corresponding to $\tilde{\mathbf{y}}$ s. The resulting vectors are represented by $\underline{\tilde{f}}_{n}$ and $\overline{\tilde{f}}_{n}$, respectively. Finally, outputs $y_{l}$ and $y_{r}$ in (6) are obtained as follows.

$$
y_{l}=\frac{\sum_{n=1}^{L} \overline{\tilde{f}}_{n} \tilde{y}^{n}+\sum_{n=L+1}^{N} \underline{\tilde{f}}_{n} \tilde{y}^{n}}{\sum_{n=1}^{L} \overline{\tilde{f}}_{n}+\sum_{n=L+1}^{N} \underline{\tilde{f}}_{n}}
$$

and

$$
y_{r}=\frac{\sum_{n=1}^{R} \underline{\tilde{f}}_{n} \tilde{y}^{n}+\sum_{n=R+1}^{N} \overline{\tilde{f}}_{n} \tilde{y}^{n}}{\sum_{n=1}^{R} \underline{\tilde{f}}_{n}+\sum_{n=R+1}^{N} \overline{\tilde{f}}_{n}}
$$


where $L$ and $R$ are computed using several iterations of EKM. Detailed step by step procedure of EKM model is presented in in [21] and [22]. The final crisp value of IT2FLS is obtained using the mean of $y_{l}$ and $y_{r}$ as follows.

$$
y=\frac{\left(y_{l}+y_{r}\right)}{2}
$$

\section{A. Basic Non-iterative Optimization Algorithm}

Type-2 fuzzy extreme learning algorithm (T2FELA) is a successful non-iterated algorithm designed for SLFNs using interval type-2 fuzzy premise parts [23]. This algorithm is summarized as follows.

For a training tuple in the form of $\left(\mathbf{x}_{i}, \mathbf{y}_{i}\right)_{i=1}^{D} \subset R^{d} \times$ $R^{m}$, an arbitrary SLFN with $\tilde{N}$ number of hidden nodes is represented as follows.

$$
\begin{aligned}
\sum_{j=1}^{\tilde{N}} \beta_{j, k} g_{j}\left(\mathbf{x}_{i}\right)=\sum_{j=1}^{\tilde{N}} \beta_{j} g\left(\mathbf{x}_{i} ; \mathbf{w}_{j}, b_{j}\right)=\mathbf{y}_{i, k}, i & =1, \cdots, D \\
k & =1, \cdots, m
\end{aligned}
$$

where $g\left(\mathbf{x}_{i} ; \mathbf{w}_{j}, b_{j}\right)$ is the activation function acting on the hidden layer and $\mathbf{w}_{j}$ and $b_{j}$ are its parameters. The prediction error is considered as follows:

$$
\begin{aligned}
e_{i k}=\mathbf{y}_{i, k}-\sum_{j=1}^{\tilde{N}} \beta_{j, k} g_{j}\left(\mathbf{x}_{i}\right) \quad i & =1, \cdots, D \\
k & =1, \cdots, m
\end{aligned}
$$

\section{Modified Non-Iterative Training BASED ON DYNAMIC COST FUNCTION}

A discrete time dynamic relationship between current and past error is defined as follows:

$$
\begin{aligned}
s_{i k}=e_{i k}+\lambda e_{(i-1) k} \quad & =1, \cdots, D \\
k & =1, \cdots, m
\end{aligned}
$$

where $\lambda>0$ is a design parameter which is chosen as $|\lambda|<1$ to guarantee the stability of the dynamic surface. The equation (11) can be represented in the following matrix form:

$$
\mathbf{H}_{2, D} \boldsymbol{\beta}+\lambda \mathbf{H}_{1, D-1} \boldsymbol{\beta}=\mathbf{Y}_{2, D}+\lambda \mathbf{Y}_{1, D-1}
$$

where

$$
\begin{array}{r}
\mathbf{H}_{i, j}\left(\mathbf{x}_{i}, \cdots, \mathbf{x}_{j} ; \mathbf{w}_{1}, \cdots, \mathbf{w}_{\tilde{N}}, b_{1}, \cdots, b_{\tilde{N}}\right) \\
=\left[\begin{array}{ccc}
g\left(\mathbf{x}_{i} ; \mathbf{w}_{1}, b_{1}\right) & \cdots & g\left(\mathbf{x}_{i} ; \mathbf{w}_{\tilde{N}}, b_{\tilde{N}}\right) \\
\vdots & \ddots & \vdots \\
g\left(\mathbf{x}_{j} ; \mathbf{w}_{1}, b_{1}\right) & \cdots & g\left(\mathbf{x}_{j} ; \mathbf{w}_{\tilde{N}}, b_{\tilde{N}}\right)
\end{array}\right]_{(i-(j+1)) \times \tilde{N}} \\
\boldsymbol{\beta}=\left[\begin{array}{c}
\boldsymbol{\beta}_{1}^{T} \\
\vdots \\
\boldsymbol{\beta}_{\tilde{N}}^{T}
\end{array}\right]_{\tilde{N} \times m}=\left[\begin{array}{ccc}
\beta_{1}^{1} & \cdots & \beta_{1}^{m} \\
\cdots & \ddots & \cdots \\
\beta_{\tilde{N}}^{1} & \cdots & \beta_{\tilde{N}}^{m}
\end{array}\right]_{\tilde{N} \times m}
\end{array}
$$

and

$$
\mathbf{Y}_{i, j}=\left[\begin{array}{c}
\mathbf{y}_{i}^{T} \\
\vdots \\
\mathbf{y}_{j}^{T}
\end{array}\right]_{(j-(i+1)) \times m}
$$

where $\mathbf{H}$ is a matrix composed of the outputs of hidden layer; generated randomly with parameters $\mathbf{w}_{j}$ and $b_{j} . \boldsymbol{\beta}$ is the output weight matrix and $\mathbf{y}^{T}$ represent the transpose of vector $\mathbf{y}$. The parameter $\widehat{\boldsymbol{\beta}}$, the optimal values of $\boldsymbol{\beta}$ is obtained as the solution to (12) as follows.

$$
\widehat{\boldsymbol{\beta}}=\left(\mathbf{H}_{2, D}+\lambda \mathbf{H}_{1, D-1}\right)^{\dagger}\left(\mathbf{Y}_{2, D}+\lambda \mathbf{Y}_{1, D-1}\right)
$$

where $\mathbf{H}^{\dagger}$ is the Moore-Penrose generalized inverse of matrix $\mathbf{H}$ [24] and [25].

The proposed parameter update rule based on (16) is presented in Fig. 2.

Remark 1. Since the most important novelty of the present paper lies in its novel cost function, this cost function needs to be justified in more details. In the case this cost function made equal to zero, a relationship between the past and current error is satisfied. In this relationship, the current error is equal to the past error when it is multiplied by a value $\lambda$ which is less than one. This means that the error has a decreasing value which may result in more stability for the training phase.

Remark 2. It is important to note that although the parameter $\lambda$ adds more degrees of freedom, its selection may be challenging and may require trial and error. Based on our experiments, an appropriate selection of this parameter would be $\lambda=-0.5$. However, the tunning of this parameter may result in superior performance.

\section{Simulation Results}

\section{A. Performance indexes}

The proposed algorithm is tested according to the most common performance indexes namely mean absolute percentage error (MAPE), adopted mean absolute percentage error (AMAPE) [26], symmetric mean absolute percentage error (SMAPE) and root mean squared error (RMSE).

$$
\begin{gathered}
M A P E=\frac{1}{N} \sum_{t=1}^{N}\left|\frac{A_{t}-F_{t}}{A_{t}}\right| \times 100 \\
A M A P E=\frac{1}{N} \sum_{t=1}^{N}\left|\frac{A_{t}-F_{t}}{\frac{1}{N} \sum_{t=1}^{N} A_{t}}\right| \times 100 \\
R M S E=\sqrt{\frac{1}{N} \sum_{t=1}^{N}\left(A_{t}-F_{t}\right)^{2}}
\end{gathered}
$$

where $N$ represent test sample number. The parameters $A_{t}$ and $F_{t}$ represent actual and foretasted values at time instance $t$.

Symmetric mean absolute percentage error (SMAPE) is an error indicator based on the relationship between absolute error and the average value of real and predicted values. This performance index is represented as follows: 


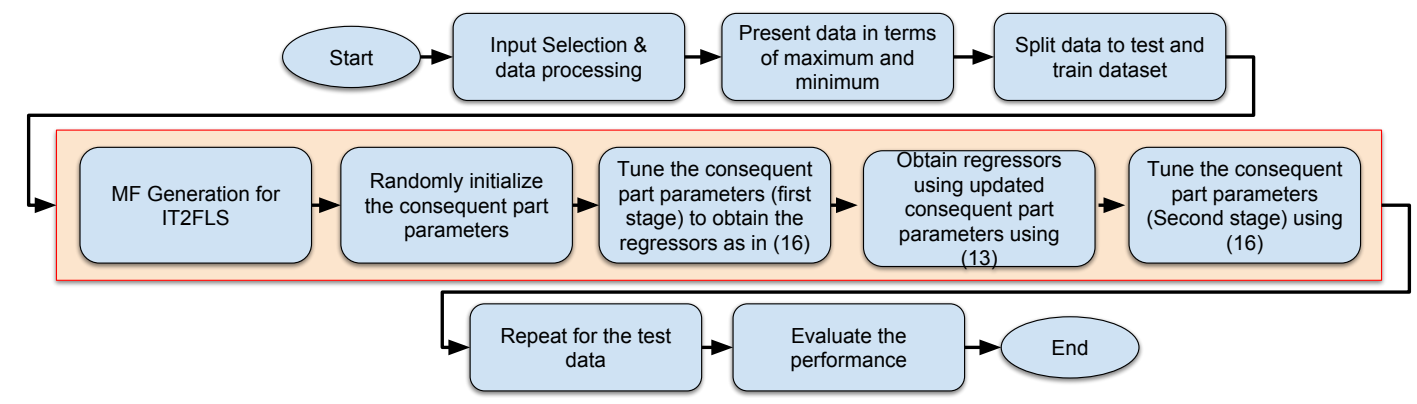

Fig. 2: Sequential diagram of the proposed training algorithm

$$
\text { SMAPE }=\frac{1}{N} \sum_{t=1}^{n} \frac{\left|F_{t}-A_{t}\right|}{\left(\left|A_{t}\right|+\left|F_{t}\right|\right) / 2} \times 100
$$

where $N, A_{t}$ and $F_{t}$ are defined in the same way as in (19).

\section{B. Prediction of the SGD/USD exchange rate}

Cross border activities and foreign exchange market require the prediction of exchange rate between major currencies across the globe. Accurate forecast of the exchange rate results in considerable decrease in risks and makes the overall financial activity profitable. Singapore Dollar as one of the major currencies is chosen here to test the prediction accuracy of the proposed approach when it comes to financial data. The data is chosen from $3-J a n-1994$ to $29-A p r-2016$. Totally, the number of entries in investigated data set is equal to 5735 samples. The missing data appears in the dataset as a result of holidays, in which the latest exchange rate is used to fill the missing data entry. The data samples from 3-Jan-1994 till $14-J a n-2005$ are used for training and the data samples from $15-$ Jan - 2005 till $29-A p r-2016$ are used for testing of the proposed method.

Six individual fuzzy rules are used to predict the financial data with their center and sigma variables being completely random variables. The data set is normalized between zero and one and the centers are uniformly distributed in this interval. The sigma values are chosen uniformly from the interval of $[0.2,0.5]$. In order to have statistically meaningful results, the experiments are repeated for 30 times and the mean values are reported in the Table I. As can be seen from this table, performance of the proposed method is better than T2FELA considerably. Moreover, the prediction performance for test data is illustrated in Fig. 3a. This figure shows that it is possible to use the data points between $3-\mathrm{Jan}-1994$ and $14-$ Jan -2005 and the trained system is capable of predicting the exchange rate of $15-$ Jan - 2005 till $29-$ $A p r-2016$. Figure $3 \mathrm{~b}$ depicts that the graph of identified data with respect to real data is very close to $y=x$ line.

As mentioned earlier, the reason behind better performance of the proposed algorithm over T2FELA is that the addition of a parameter to the cost function makes it more stable. In this case, the mean value of condition number for the proposed method is obtained as $1.8619 \times 10^{6}$ while it is being equal to $1.1345 \times 10^{7}$ for T2FELA. Similar to the previous case, this fact clearly shows the reason why
TABLE I: Results of comparison between the proposed SMC based non-iterative optimization method and T2FELA.

\begin{tabular}{|l|c|c|c|c|}
\hline & \multicolumn{1}{|c|}{ Test } & Data & \multicolumn{2}{c|}{ Train } \\
\hline Index & $\begin{array}{c}\text { The proposed } \\
\text { method }\end{array}$ & T2FELA & $\begin{array}{c}\text { The proposed } \\
\text { method }\end{array}$ & T2FELA \\
\hline MAPE \% & 0.4325 & 0.7353 & 0.2505 & 0.4715 \\
AMAPE \% & 0.4477 & 0.7561 & 0.2465 & 0.4642 \\
RMSE & 0.0044 & 0.0074 & 0.0024 & 0.0045 \\
SMAPE \% & 0.4342 & 0.7374 & 0.2505 & 0.4715 \\
\hline
\end{tabular}

the results obtained by the proposed method outperform the results obtained by T2FELA.

\section{Prediction of the Euro/USD exchange rate}

In this section, the proposed non-iterative algorithm is used to predict the daily exchange rate of Euro versus USD for 4558 days in the interval of $30-$ Oct - 1998 to $15-A u g-2016$. For missing data resulted from holidays or unavailable data samples, the latest exchange rate is used. The data samples from $30-O c t-1998$ till $8-A u g-2007$ is used for training and the data samples from $9-A u g-2007$ till 15-Aug-2016 are used for testing the proposed method.

Four rules are considered for the fuzzy system with completely random center and sigma variables. For the prediction purpose, the exchange rates are normalized to the interval of $[0,1]$ and the centers are uniformly distributed in this interval. However, the indices are all evaluated based on the true data. The sigma values are chosen uniformly from the interval of $[0.2,0.5]$. Similar to the previous case, Table II illustrates that the proposed method outperforms T2FELA considerably. In order to have statistically meaningful results, the experiments are repeated for 30 times and the mean values are reported in the table. Moreover, the prediction performance for test data is presented in Fig. 4a. Moreover, Figure $4 \mathrm{c}$ shows how identification error is distributed around zero. Figure $4 \mathrm{~d}$ depicts that the graph of identified data with respect to real data is very close to $y=x$ line.

Similarly, the authors believe that the reason behind better performance of the proposed algorithm over T2FELA is the proposed method is numerically more stable. The fact that the mean value of condition number for the proposed method is obtained as $3.5774 \times 10^{8}$ while it is being equal to $1.9220 \times$ $10^{9}$ for T2FELA supports this idea. 


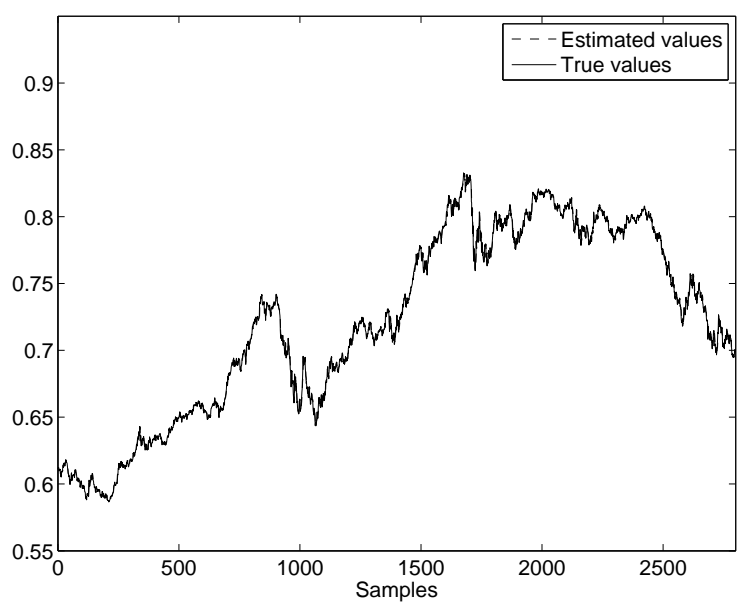

(a) Testing performance of the proposed algorithm for the prediction of SGD/USD exchange rate

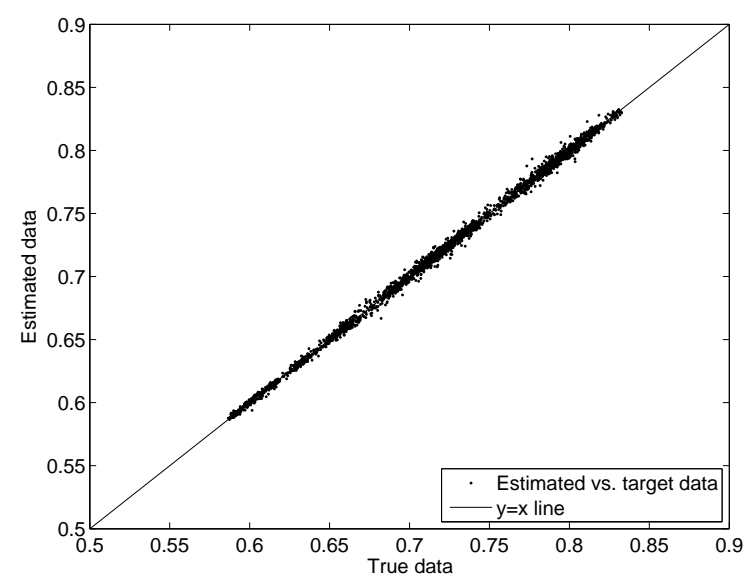

(b) Estimated values versus real values for the prediction of SGD/USD exchange rate

Fig. 3: Estimated values versus real values for the prediction of SGD/USD exchange rate

\section{CONCLusion}

The use of dynamic cost function in this paper results in overcoming the numerical problems caused by matrix inversion needed to estimate the parameters of the consequent part of IT2FLSs when using the T2FELA. Using the proposed dynamic cost function, a novel formula is extracted for the consequent part parameters of IT2FLS. Since the proposed cost function benefits from a parameter, it has more degrees of freedom which makes it possible to obtain a more numerically stable parameter update rule which results in better approximation of the true values. The proposed method is applied to predict financial datasets. Having a good prediction is required for decreasing the risk of decisions. This is the main reason why a foreign exchange dataset is selected in this study to test the prediction capability of the proposed approach. As can be inferred from the simulation results, the proposed method makes the condition number for the matrix inversion in the estimation of the parameters of the consequent part of IT2FNN closer to one, which is highly
TABLE II: Results of comparison between the proposed SMC based non-iterative optimization method and T2FELA.

\begin{tabular}{|l|c|c|c|c|}
\hline & \multicolumn{2}{|c|}{ Test } & Data & \multicolumn{2}{c|}{ Train } & Data \\
\hline Index & $\begin{array}{c}\text { The proposed } \\
\text { method }\end{array}$ & T2FELA & $\begin{array}{c}\text { The proposed } \\
\text { method }\end{array}$ & T2FELA \\
\hline MAPE \% & 0.8009 & 1.4422 & 0.5316 & 0.9952 \\
AMAPE \% & 0.8400 & 1.5065 & 0.5198 & 0.9730 \\
RMSE & 0.0189 & 0.0332 & 0.0073 & 0.0139 \\
SMAPE \% & 0.8088 & 1.4541 & 0.5316 & 0.9953 \\
\hline
\end{tabular}

desirable. Furthermore, the proposed algorithm shown to be capable of outperforming T2FELA if appropriate parameter values are selected for this algorithm.

\section{REFERENCES}

[1] J. M. Mendel, "Uncertain rule-based fuzzy logic system: introduction and new directions," 2001.

[2] Y. Liu, J. Yi, R. Yuan, and Z. Pu, "Adaptive type-2 fuzzy control for flexible air-breathing hypersonic vehicles with measurement noises," in 2018 Annual American Control Conference (ACC). IEEE, 2018, pp. $1485-1490$.

[3] E. Kayacan and M. A. Khanesar, Fuzzy Neural Networks for Real Time Control Applications: Concepts, Modeling and Algorithms for Fast Learning. Butterworth-Heinemann, 2015.

[4] E. Kayacan, O. Kaynak, R. Abiyev, J. Tørresen, M. Høvin, and K. Glette, "Design of an adaptive interval type-2 fuzzy logic controller for the position control of a servo system with an intelligent sensor," in international conference on fuzzy systems. IEEE, 2010, pp. 1-8.

[5] E. Kayacan, E. Kayacan, H. Ramon, and W. Saeys, "Towards agrobots: Identification of the yaw dynamics and trajectory tracking of an autonomous tractor," Computers and Electronics in Agriculture, vol. 115, pp. 78-87, 2015.

[6] M. A. Khanesar, E. Kayacan, M. Teshnehlab, and O. Kaynak, "Levenberg marquardt algorithm for the training of type-2 fuzzy neuro systems with a novel type-2 fuzzy membership function," in 2011 IEEE symposium on advances in type-2 fuzzy logic systems (T2FUZZ). IEEE, 2011, pp. 88-93.

[7] Q.-Y. Zhu, A. Qin, P. Suganthan, and G.-B. Huang, "Evolutionary extreme learning machine," Pattern Recognition, vol. 38, no. 10, pp. 1759 - 1763, 2005.

[8] G.-B. Huang, Q.-Y. Zhu, and C.-K. Siew, "Extreme learning machine: Theory and applications," Neurocomputing, vol. 70, no. 1-3, pp. 489 - 501, 2006.

[9] S. Hassan, M. A. Khanesar, E. Kayacan, J. Jaafar, and A. Khosravi, "Optimal design of adaptive type-2 neuro-fuzzy systems: A review," Applied Soft Computing, vol. 44, pp. 134-143, 2016.

[10] G.-B. Huang, E. Cambria, K.-A. Toh, B. Widrow, and Z. Xu, "New trends of learning in computational intelligence [guest editorial]," IEEE Computational Intelligence Magazine, vol. 10, no. 2, pp. 1617, 2015.

[11] Z. Liouane, T. Lemlouma, P. Roose, F. Weis, and H. Messaoud, "An improved extreme learning machine model for the prediction of human scenarios in smart homes," Applied Intelligence, pp. 1-14, 2017.

[12] X. Zhu, Z. Ni, M. Cheng, F. Jin, J. Li, and G. Weckman, "Selective ensemble based on extreme learning machine and improved discrete artificial fish swarm algorithm for haze forecast," Applied Intelligence, pp. 1-19, 2017.

[13] M. FernAndez-Delgado, E. Cernadas, S. Barro, J. Ribeiro, and J. Neves, "Direct kernel perceptron (dkp): Ultra-fast kernel elmbased classification with non-iterative closed-form weight calculation," Neural Networks, vol. 50, pp. 60 - 71, 2014.

[14] Y. Miche, A. Sorjamaa, P. Bas, O. Simula, C. Jutten, and A. Lendasse, "OP-ELM: Optimally pruned extreme learning machine," Neural Networks, IEEE Transactions on, vol. 21, no. 1, pp. 158-162, 2010.

[15] Y. Yang, Y. Wang, and X. Yuan, "Bidirectional extreme learning machine for regression problem and its learning effectiveness," Neural Networks and Learning Systems, IEEE Transactions on, vol. 23, no. 9, pp. 1498-1505, 2012. 

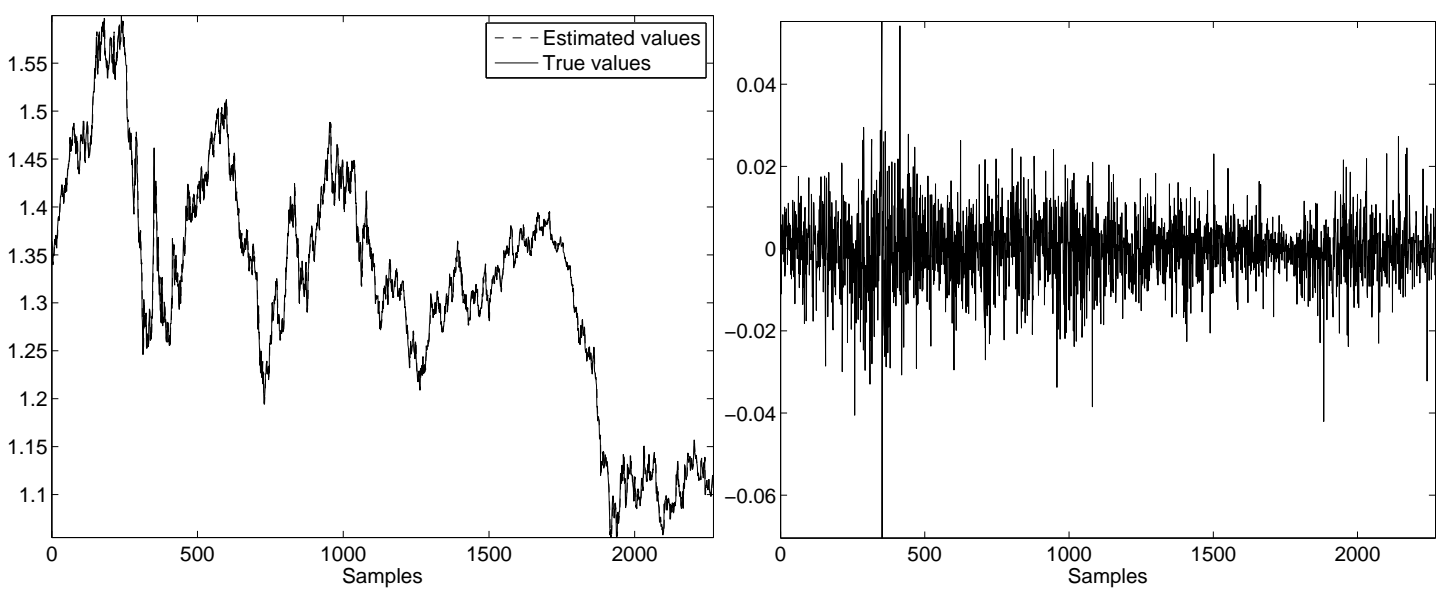

(a) Testing performance of the proposed algorithm on Euro/USD(b) Error of the proposed algorithm on Euro/USD exchange rate exchange rate
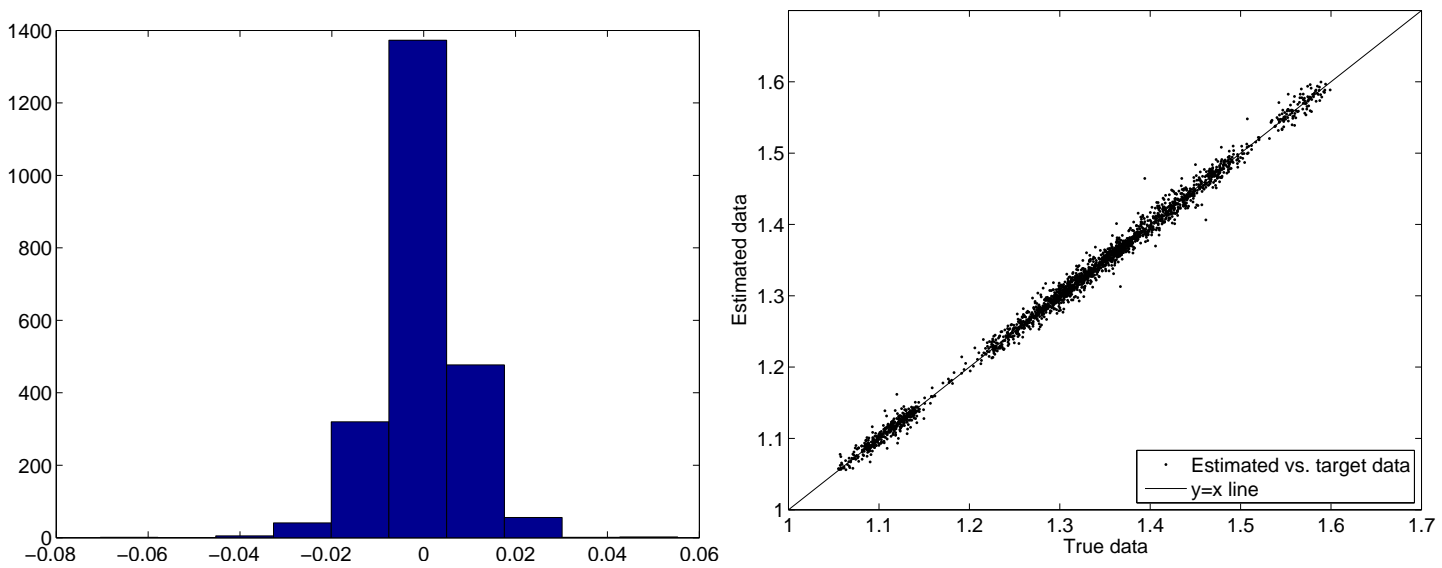

(c) Histogram of the error of the algorithm on Euro/USD(d) Estimated values versus real values on Euro/USD exchange exchange rate

rate

Fig. 4: Prediction of Euro/USD exchange rate

[16] N. Jafarpisheh and M. Teshnehlab, "Cancers classification based on deep neural networks and emotional learning approach," IET Systems Biology, 2018

[17] C.-F. Juang and Y.-W. Tsao, "A self-evolving interval type-2 fuzzy neural network with online structure and parameter learning," Fuzzy Systems, IEEE Transactions on, vol. 16, no. 6, pp. 1411-1424, Dec 2008.

[18] Q. Liang and J. Mendel, "Interval type-2 fuzzy logic systems: theory and design," Fuzzy Systems, IEEE Transactions on, vol. 8, no. 5, pp. 535-550, 2000.

[19] D. Wu, "Approaches for reducing the computational cost of interval type-2 fuzzy logic systems: overview and comparisons," Fuzzy Systems, IEEE Transactions on, vol. 21, no. 1, pp. 80-99, 2013.

[20] D. Wu and J. M. Mendel, "Enhanced karnik-mendel algorithms," Fuzzy Systems, IEEE Transactions on, vol. 17, no. 4, pp. 923-934,

[24] D. Serre, Matrices: Theory and Application. New York, USA: Springer-Verlag, 2002.
2009.

[21] N. N. Karnik and J. M. Mendel, "Centroid of a type-2 fuzzy set," Information Sciences, vol. 132, no. 1-4, pp. 195 - 220, 2001.

[22] J. M. Mendel, Uncertain Rule-Based Fuzzy Logic Systems: Introduction and New Directions. Prentice Hall, Englewood Cliffs, NJ, 2001.

[23] Z. Deng, K.-S. Choi, L. Cao, and S. Wang, "T2fela: Type-2 fuzzy extreme learning algorithm for fast training of interval type-2 tsk fuzzy logic system," Neural Networks and Learning Systems, IEEE Transactions on, vol. 25, no. 4, pp. 664-676, April 2014.

[25] C. Rao and S. Mitra, Generalized Inverse of Matrices and its Applications. Wiley, New York, ., 1971.

[26] P. Mandal, T. Senjyu, N. Urasaki, T. Funabashi, and A. Srivastava, "A novel approach to forecast electricity price for pjm using neural network and similar days method," Power Systems, IEEE Transactions on, vol. 22, no. 4, pp. 2058-2065, Nov 2007. 